\title{
Fisetin Attenuates Lipopolysaccharide-Induced Inflammatory Responses in Macrophage
}

\author{
Yoshiko Hada, ${ }^{1,2}$ Haruhito A. Uchida ${ }^{10}{ }^{1,2}$ and Jun Wada ${ }^{1}$ \\ ${ }^{1}$ Department of Nephrology, Rheumatology, Endocrinology and Metabolism, Okayama University Graduate School of Medicine, \\ Dentistry and Pharmaceutical Science, Okayama, Japan \\ ${ }^{2}$ Department of Chronic Kidney Disease and Cardiovascular Disease, Okayama University Graduate School of Medicine, \\ Dentistry and Pharmaceutical Science, Okayama, Japan
}

Correspondence should be addressed to Haruhito A. Uchida; hauchida@okayama-u.ac.jp

Received 27 January 2021; Revised 23 March 2021; Accepted 31 March 2021; Published 13 April 2021

Academic Editor: Tanveer A. Wani

Copyright (c) 2021 Yoshiko Hada et al. This is an open access article distributed under the Creative Commons Attribution License, which permits unrestricted use, distribution, and reproduction in any medium, provided the original work is properly cited.

\begin{abstract}
Several studies have reported the efficacy and safety of polyphenols in human health; however, the verification of their efficacy remains insufficient. The aim of this study was to examine whether fisetin, one of flavonoids prevalently present in fruits and vegetables, could suppress lipopolysaccharide- (LPS-) induced inflammatory responses in macrophages. LPS increased proinflammatory mRNA abundance (MCP 1, IL-1 $\beta$, and iNOS) but were suppressed by fisetin. The increment of nitric oxide by LPS, an oxidative stress factor, was attenuated by fisetin. In addition, LPS-enhanced phosphorylation of mitogen-activated protein kinase (ERK and JNK) was reduced. Finally, fisetin attenuated the expression or activity of uPA, uPAR, MMP-2, and MMP-9, which are known as associated factors of macrophage recruitment or infiltration. In conclusion, fisetin is a promising therapeutic agent for macrophage-related inflammation diseases, like sepsis and atherosclerosis.
\end{abstract}

\section{Introduction}

The therapeutic usage of natural components has gained more attention in the last 2 decades. Based on their great efficacy and low toxicity, 40\% of FDA-approved therapeutic agents are natural-based components or their derivatives [1]. Toxicity associated with other drugs has been successfully prevented by different natural products [2]. Thus, it is very important and significant to explore plant-derived natural compounds further.

Polyphenols are the plant secondary metabolites and widely present in foods and beverages of plant origin, fruits, vegetables, spices, tea, and wine. Although they are not considered as essential micronutrients, several literatures evince their beneficial effects on human health, especially in diets associated with high consumption of fruits and vegetables [3-5]. Numerous studies have been performed over decades for investigation for pharmacological effects of polyphenols, suggesting benefit for health. Growing evidence has shown that a few polyphenols possess anti-inflammatory properties
[6-8]. In addition, recent studies have demonstrated that several kinds of polyphenols can inhibit regulatory enzymes or transcription factors which are involved in inflammation [9].

Diet influences different stages of inflammation and demonstrates an important impact on several inflammatory diseases $[10,11]$. Inflammation plays a key role on the development of diseases such as diabetes, asthma, cardiovascular diseases, and cancer [12]. Therefore, controlling inflammation may prevent these diseases. Since macrophages are major mediators of inflammation, they have diverse functions in tissue homeostasis and inflammation. Thus, agents effectively modulating their functions are of great clinical value since aberrant activation of macrophages can cause detrimental immune responses. Current research tendency clearly indicates that natural products are one of the most important sources of new drugs [13].

One polyphenol, fisetin $\left(3,3^{\prime}, 4^{\prime}, 7\right.$-tetrahydroxyflavone), is contained richly in strawberry, apple, tomato, onion, orange, peach, grape, kiwi, persimmon, tea, and wine [14]. So far, fisetin has reported strong anti-inflammatory [15-17], 

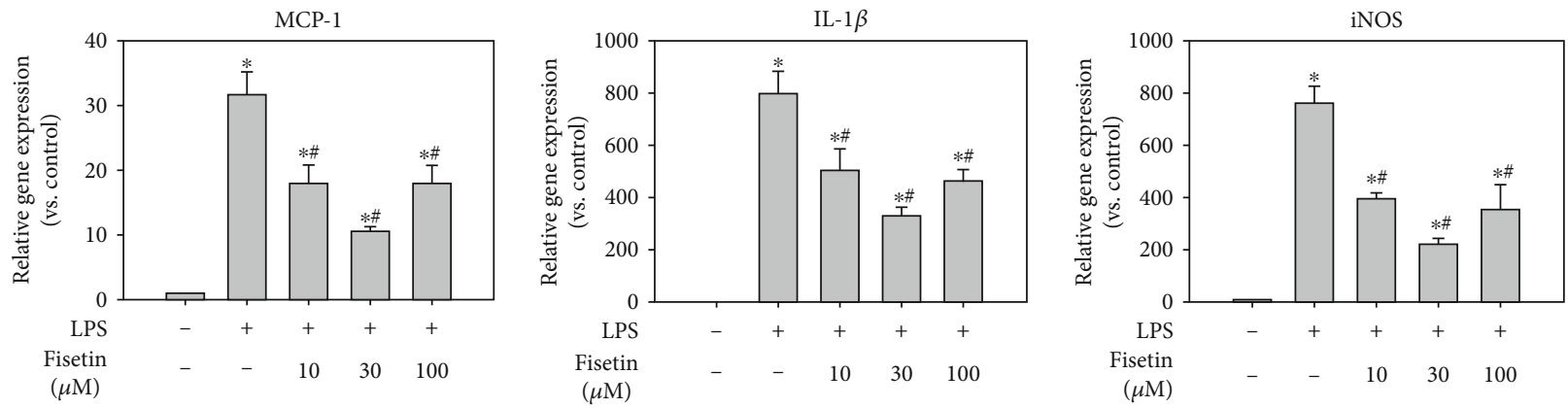

(a)

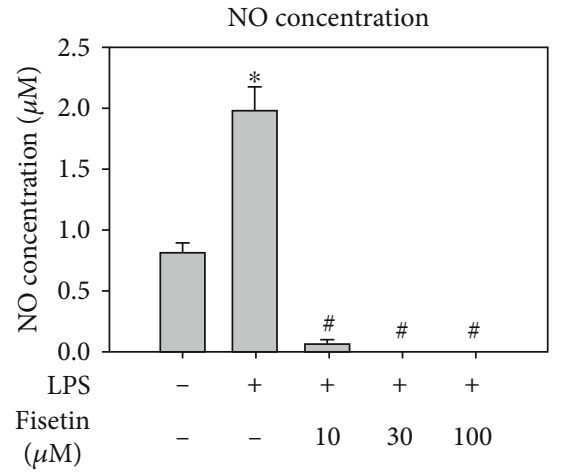

(b)

FIGURE 1: Effects of fisetin on the expression of inflammatory mediators in macrophages stimulated with LPS. (a) mRNA expression of MCP$1, \mathrm{IL}-1 \beta$, and iNOS in LPS- $(10 \mathrm{ng} / \mathrm{mL})$ stimulated macrophages in the presence or absence of fisetin $(10-100 \mu \mathrm{M})$ by qPCR $(n=6$ in each group). Each bar presents the mean + SE of six experiments. ${ }^{*} p<0.05$ vs. control group; ${ }^{*} p<0.05$ vs. LPS-stimulated group. (b) NO concentration in each cell culture supernatants by colorimetric assay ( $n=3$ in each group). Each bar presents the mean $+\mathrm{SE}$ of three experiments. ${ }^{*} p<0.05$ vs. control; ${ }^{*} p<0.05$ vs. LPS.

antioxidant [18], antitumorigenic [19, 20], antiangiogenic [21], antidiabetic [22], neuroprotective [23], and cardioprotective effects [24] both in cell culture and in animal models relevant to human diseases. Although fisetin has many physiological effects, its effect on macrophages is not much reported.

In the present study, we investigated the beneficial effects of fisetin on the inflammatory response of macrophages activated by lipopolysaccharide (LPS).

\section{Materials and Methods}

2.1. Isolation of Resident Peritoneal Macrophages. BALB/c mice were obtained from the Institute of Medical Science of the University of Tokyo and maintained in the Animal Resource Center of Okayama University. All the animal experiments were performed to conform to the NIH guidelines (Guide for the Care and Use of Laboratory Animals). The experimental protocol was approved by the Ethics Review Committees for Animal Experimentation of Okayama University Graduate School of Medicine, Dentistry, and Pharmaceutical Sciences (OKU-2018449). All mice were maintained in a barrier facility and fed a normal laboratory diet. Male and female BALB/c mice, $16 \pm 4$ weeks of age were anesthetized with isoflurane and euthanized via cervical dislocation. Macrophages were harvested via peritoneal lavage using saline $(5 \mathrm{~mL})$. The peritoneal lavage solution was cen- trifuged at $1500 \mathrm{rpm}$ for 5 minutes. The sediment was suspended in a little DMEM (SIGMA, Cat. No. D6046). Red blood cells were lysed using a solution of ammonium chloride. The number of macrophages were calculated using a hemocytometer, then resuspended in DMEM containing heat-inactivated FBS $(10 \% v / v)$, penicillin $(10 \mathrm{U} / \mathrm{mL})$, and streptomycin $(10 \mathrm{mg} / \mathrm{mL})$, and plated into 6-well plates (Corning Incorporated, Cat. No. 3516) [25, 26].

2.2. Cell Culture Conditions. After overnight serum starvation, macrophages were incubated with selected concentrations of fisetin $(10,30$, and $100 \mu \mathrm{M}$; SIGMA, Cat. No. F4043) or vehicle for 3 hours and incubated in the presence or absence of LPS (10 ng/mL; SIGMA, Cat. No. L4391) for an appropriate time. Then, the medium and cells were collected. The medium was used for the nitric oxide (NO) assay and gelatin zymography. Cell lysates were used for quantitative polymerase chain reaction (qPCR) and western blotting.

2.3. Real-Time Polymerase Chain Reaction. mRNAs were extracted from macrophages using RNeasy Mini Kits (QIAGEN, Cat. No. 74104). Reverse transcription was performed using an iScript cDNA Synthesis Kit (Bio-Rad, Cat. No. 1708891). qPCR was performed with an ABI Step One Real-Time PCR System (Applied Biosystems, QuantStudio 3) using a Fast SYBR Green Real-time PCR Mixture (Applied Biosystems, Cat. No. 4385612) [27]. Primers for monocyte 


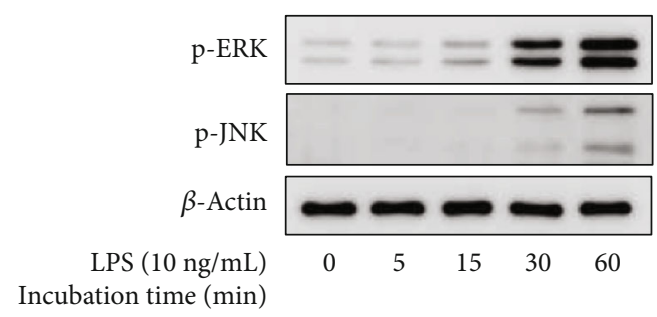

(a)

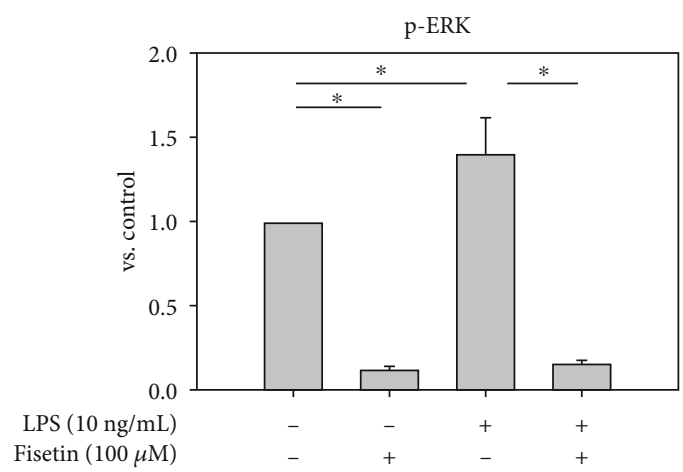

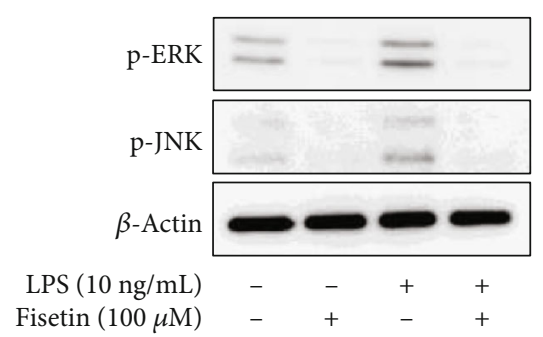

(b)

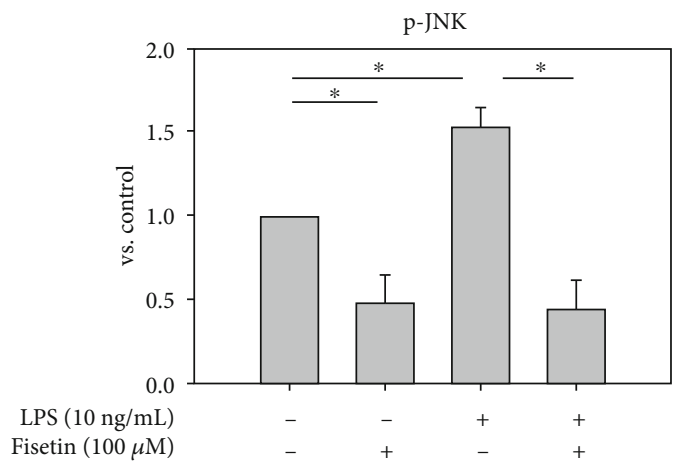

(c)

FIGURE 2: Effects of fisetin on the protein expression of MAPKs in macrophages stimulated with LPS. (a) Protein expression of MAPK in LPS$(10 \mathrm{ng} / \mathrm{mL})$ stimulated macrophages for $0-60$-minute incubation $(n=3$ in each group). (b) Protein expression of MAPK in LPS- (10 ng/mL) stimulated macrophages in the presence or absence of fisetin $(100 \mu \mathrm{M})$ by western blotting $(n=3$ in each group). (c) The relative expression of each protein was quantified by densitometry and normalized to the band under the control condition. Each bar presents the mean + SE of three experiments. ${ }^{*} p<0.05$.

chemotactic protein 1 (MCP-1) (Takara Bio Inc., Cat. No. MA066003), interleukin 1 beta (IL-1 $\beta$ ) (Takara Bio Inc., Cat. No. MA025939), inducible nitric oxide synthase (iNOS) (Takara Bio Inc., Cat. No. MA083369), matrix metalloproteinase- (MMP-) 2 (Takara Bio Inc., Cat. No. MA127110), MMP-9 (Takara Bio Inc., Cat. No. MA031311), and 18S ribosomal RNA (18S) (Takara Bio Inc., Cat. No. MA050364) were commercially available. Each sample was normalized to values for $18 \mathrm{~S}$ mRNA expression ( $\triangle \Delta \mathrm{CT}$ method) [27].

2.4. Nitric Oxide Assay. The individual cell culture supernatants were collected and centrifuged $10 \mathrm{~min}$ at $14,000 \mathrm{rpm}$ at $4^{\circ} \mathrm{C}$. Then, the supernatant was used for nitric oxide (NO) assay. The NO assay was performed as described in the commercially available kits (BioAssay Systems, Cat. No. D2NO-100). BioAssay Systems' QuantiChrom ${ }^{\mathrm{TM}}$ Nitric Oxide Assay Kit is a colorimetric assay for the determination of NO production following reduction of nitrate to nitrite using the improved Griess method.

2.5. Western Blotting. Whole cell proteins were extracted from macrophages using a lysis buffer (Cell Signaling, Cat. No. 9803). Each sample was applied into $10 \%$ SDS-PAGE and transferred to a polyvinylidene fluoride membrane, immunoblotted with primary antibodies (the phosphorylation of extracellular signal-regulated kinase (p-ERK); Cell Signaling, Cat. No. 9101, the phosphorylation of c-Jun Nterminal kinase (p-JNK); Cell Signaling, Cat. No. 9251, the urokinase plasminogen activator (uPA); Abcam, Cat. No. ab20789, the urokinase plasminogen activator and receptor (uPAR); R\&D, Cat. No. AF534, and $\beta$-actin; Sigma Cat. No. A5441) [28]. Membranes were then incubated with appropriate secondary antibodies, and immune complexes were visualized on chemiluminescence (Merck Millipore, Cat. no. WBLUF0100, Cat. no. WBLUC0100) and quantified using a General Electric Imager (GE Healthcare, LAS 4000 mini) [29].

2.6. Gelatin Zymography. Cell culture supernatants were resolved under nonreducing conditions by SDS-PAGE $(10 \% \mathrm{wt} / \mathrm{vol})$ polymerized in the presence of gelatin $(0.1 \%$ $\mathrm{wt} / \mathrm{wt}$ ) to evaluate the activities of MMP-9. Gels were washed with Triton X-100 (2.5\% vol/vol) for $60 \mathrm{~min}$ and distilled water for $15 \mathrm{~min}$ two times. Gels were then incubated overnight at $37^{\circ} \mathrm{C}$ in $50 \mathrm{mM}$ Tris buffer containing calcium chloride $(5 \mathrm{mM}) \mathrm{pH}$ 8.0. After incubation, gels were stained with Coomassie Brilliant Blue R-250 (Bio-Rad, Cat. No. 161-0436) for $30 \mathrm{~min}$ at room temperature, then followed by destaining with Coomassie Brilliant Blue R-250 Destaining Solution (Bio-Rad, Cat. No. 161-0438) until clear bands appeared. Gel images were quantified using a General Electric Imager (GE Healthcare, LAS 4000 mini); the unstained, translucent digested regions represented areas of MMP activity [30].

2.7. Statistics. All statistical analyses were performed using Sigma Plot v14.0 (Systat Software Inc., California, USA). Data are presented as the mean \pm standard error of the mean. Statistical significance between multiple groups was assessed by one-way or two-way analysis of variance followed 

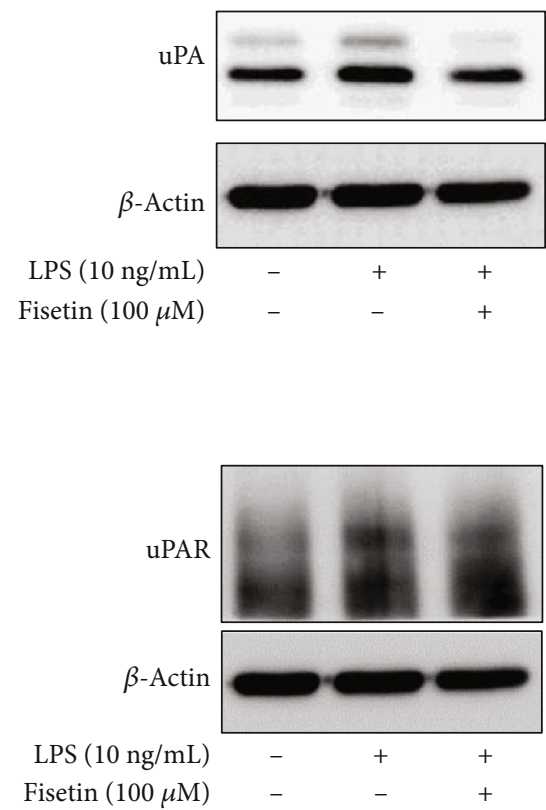

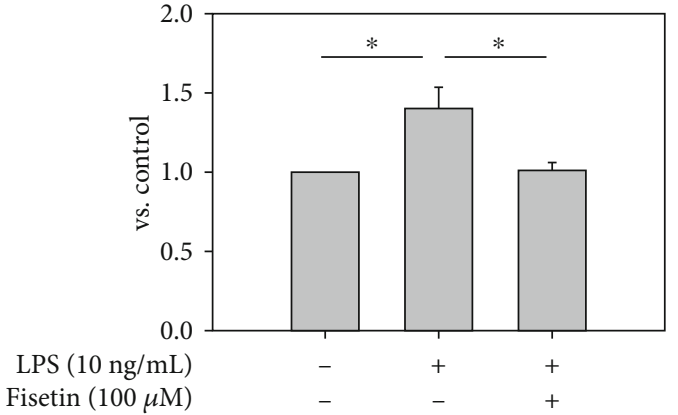

(a)

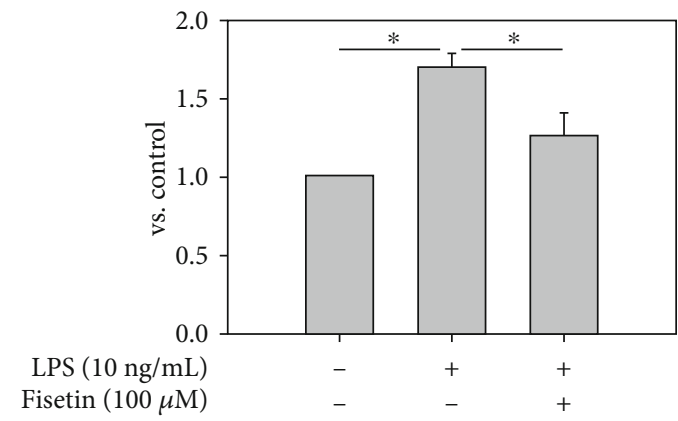

(b)

FIGURE 3: Effects of fisetin on the protein expression of uPA and uPAR in macrophages stimulated with LPS. (a) Protein expression of uPA in LPS- $(10 \mathrm{ng} / \mathrm{mL})$ stimulated macrophages in the presence or absence of fisetin $(100 \mu \mathrm{M})$ by western blotting. The relative expression of each protein was quantified by densitometry and normalized to the band under the control condition ( $n=3$ in each group). Each bar presents the mean + SE of three experiments. ${ }^{*} p<0.05$. (b) Protein expression of uPAR in LPS- $(10 \mathrm{ng} / \mathrm{mL})$ stimulated macrophages in the presence or absence of fisetin $(100 \mu \mathrm{M})$ by western blotting. The relative expression of each protein was quantified by densitometry and normalized to the band under the control condition ( $n=3$ in each group). Each bar presents the mean + SE of three experiments. ${ }^{*} p<0.05$.

by Holm-Sidak post hoc test or Student-Newman-Keuls post hoc test. $p$ value $<0.05$ was considered statistically significant.

\section{Results}

3.1. Fisetin Attenuated Inflammatory Mediators in Macrophages Stimulated with LPS. Since activated macrophages produce proinflammatory cytokines and inflammatory mediators such as NO [8], we examined the effects of fisetin on inflammatory mediators in macrophages stimulated with LPS. First, to study whether fisetin exerts the antiinflammatory effect at the transcriptional level, we determined mRNA expression levels of inflammatory genes such as MCP1 , IL-1 $\beta$, and iNOS (Figure 1(a)). As expected, the expressions of these inflammatory genes were suppressed by fisetin in a dose-dependent manner. Next, to confirm whether fisetin suppresses inflammatory response in macrophages, we evaluated its capability to suppress NO production in LPS-treated macrophages (Figure 1(b)). As expected, fisetin decreased production of NO in a dose-dependent manner.

3.2. Fisetin Reduced Mitogen-Activated Protein Kinase in Macrophages Stimulated with LPS. Since fisetin was reported to inhibit mitogen-activated protein kinase (MAPK) pathways in various cell types [31,32], we examined the effects of fisetin on MAPKs in macrophages. First, activation of MAPKs induced by LPS in macrophages was observed
(Figure 2(a)). The increase in p-ERK by LPS peaked at 15 minutes after stimulation, while the increment of p-JNK by LPS peaked at 30 minutes. Thus, in the subsequent experiments, macrophages were stimulated with LPS for 30 minutes. Cotreatment with fisetin significantly decreased LPS-induced enhancement of p-ERK and p-JNK (Figures 2(b) and 2(c)).

3.3. Fisetin Reduced $u P A$ and $U P A R$ in Macrophages Stimulated with LPS. Macrophage activation is closely correlated to the invasiveness of cells. In addition, uPA and UPAR in macrophages contribute to the infiltration of cells. Thus, the effect of fisetin on the expression of uPA and uPAR was investigated. LPS induced the protein abundance of uPA (Figure 3(a)) and uPAR (Figure 3(b)). Cotreatment of fisetin reduced the increment of uPA (Figure 3(a)) and UPAR by LPS (Figure 3(b)).

3.4. Fisetin Reduced MMP-2 and MMP-9 in Macrophages Stimulated with LPS. Furthermore, to elucidate effect of fisetin on MMPs, we examined MMP-2 and MMP-9 expressions, the downstream mediators of UPA/uPAR. Regarding the mRNA level, LPS significantly increased MMP-2 and MMP-9 mRNA expression, compared with the vehicle treatment (Figure 4(a)). Fisetin reduced the enhancement of these gene expressions of MMP-2 or MMP9 induced by LPS in a dose-dependent manner (Figure 4(a)). Moreover, the protein activities of MMP-9 were evaluated by zymography 

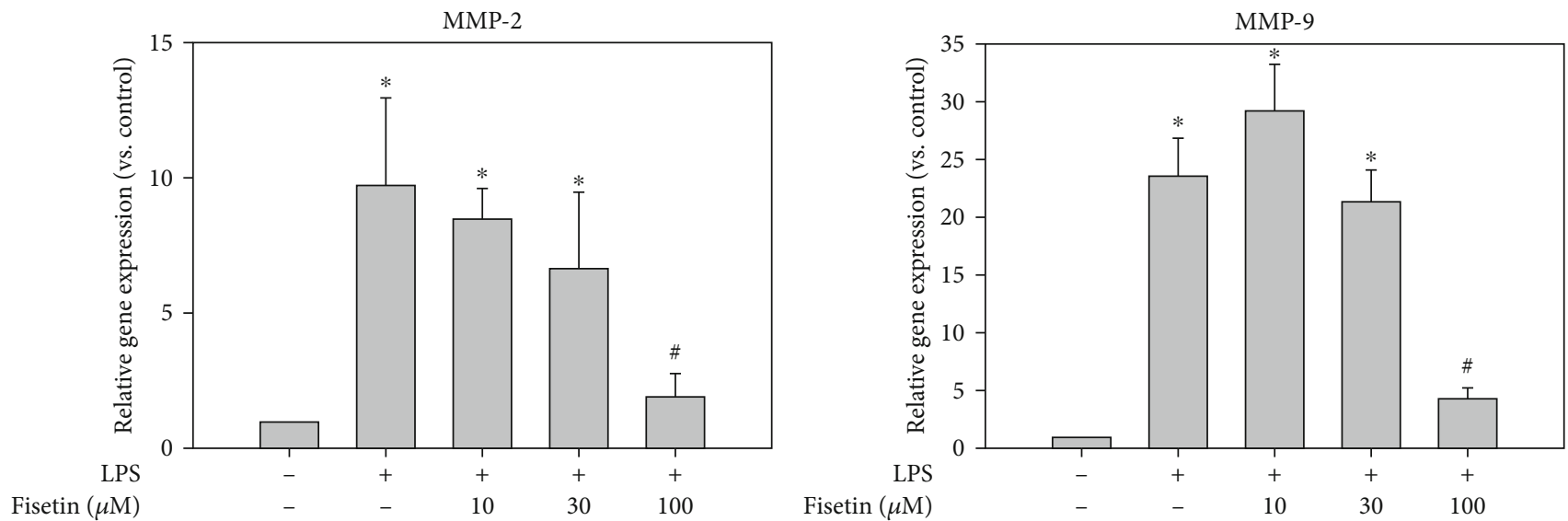

(a)
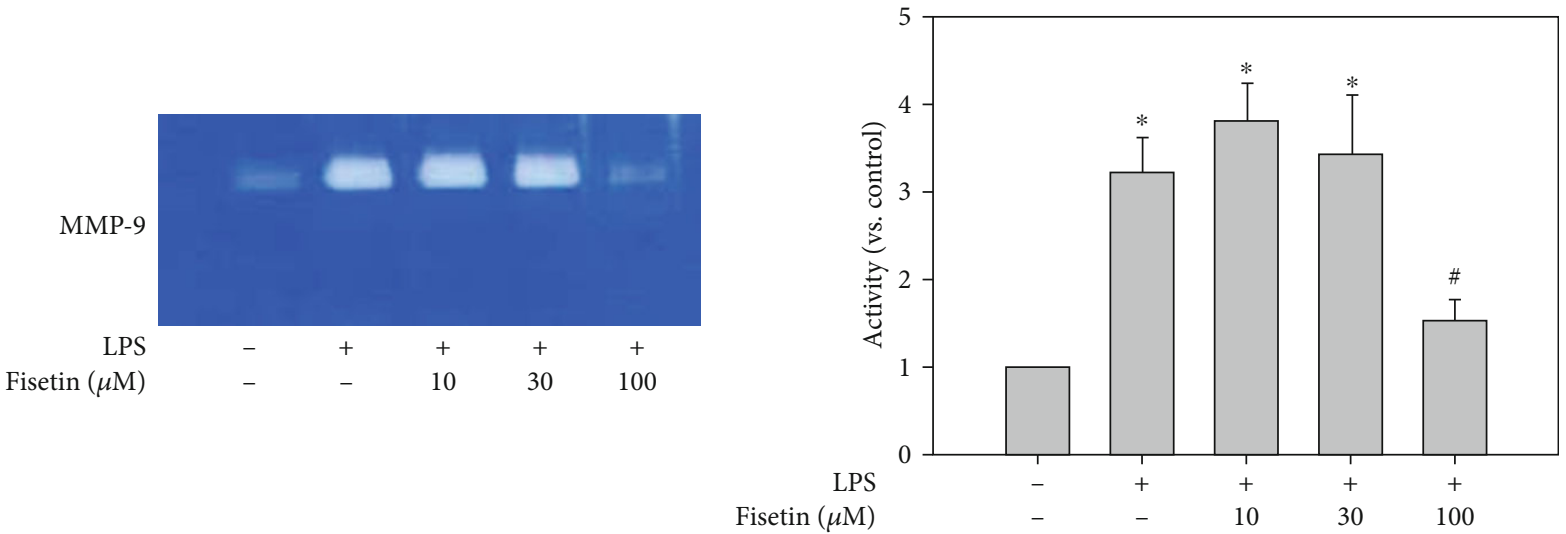

(b)

FIGURE 4: Effects of fisetin on MMP-2 and MMP-9 in macrophages stimulated with LPS. (a) mRNA expression of MMP-2 and MMP-9 in LPS- $(10 \mathrm{ng} / \mathrm{mL})$ stimulated macrophages in the presence or absence of fisetin $(10-100 \mu \mathrm{M})$ by qPCR ( $n=6$ in each group). Each bar presents the mean + SE of six experiments. ${ }^{*} p<0.05$ vs. control group; ${ }^{*} p<0.05$ vs. LPS-stimulated group. (b) The activation of MMP-9 in LPS- $(10 \mathrm{ng} / \mathrm{mL})$ stimulated macrophages in the presence or absence of fisetin $(10-100 \mu \mathrm{M})$ by gelatin zymography. The relative expression of MMP-9 activity was quantified by densitometry and normalized to the band under the control condition $(n=3$ in each group). Each bar presents the mean + SE of three experiments. ${ }^{*} p<0.05$.

(Figure 4(b)). The protein activities of MMP-9 $(92 \mathrm{kDa})$ were increased by LPS stimulation, which was reduced by fisetin (Figure 4(b)).

\section{Discussion}

We found an important effect of fisetin against inflammation in macrophages. The present study demonstrated that fisetin attenuated the induction of inflammatory components such as MCP-1, IL- $1 \beta$, iNOS, and NO, induced by LPS in macrophages. In addition, fisetin suppressed the activation of MAPK and attenuated the LPS-induced increment of uPA and UPAR expression. Moreover, fisetin attenuated the increase of mRNA abundance of MMP-2 and MMP-9 and the activity of MMP-9.

In sepsis caused by gram-negative infection, LPS is commonly recognized as the causative agent of sepsis. In many studies, LPS has been used as a model of sepsis as well as an inflammation. Moreover, chronic inflammation induces early cancer by activation of inflammatory cells and improper production of a preinflammatory mediator like iNOS and tran- scription factors like the nuclear transcription factor kappa B (NF- $\kappa \mathrm{B}$ ) [33]. Besides, it has also been reported that oxidative stress is reduced by attenuating the expression of iNOS [34]. Our study demonstrated that fisetin may be effective in the prevention and treatment of sepsis and septic shock, due to the suppressive effect on mRNA abundance of proinflammatory genes and the production of NO. In this study, fisetin suppressed NO production by reducing the expression of mRNA of the proinflammatory gene (iNOS); therefore, it was likely that fisetin attenuated the oxidative stress. Consequently, these results supported the anti-inflammatory, antitumorigenic, and antioxidant effects of fisetin.

Several studies have reported that LPS induced the phosphorylation of MAPKs, including ERK and JNK [35]. In addition, it has been reported that fisetin inhibits migration and invasion of human cervical cells by downregulating uPA expression through suppressing MAPK [36]. In macrophages, few reports are available regarding the effect of fisetin on the expression and activity of uPA and uPAR. Our study demonstrated for the first time that fisetin suppressed the expression of uPA and uPAR induced by LPS through 
suppressing MAPK signaling in macrophages, as previously reported in cancer cells.

MMPs are a family of proteases whose expression is related to certain processes, such as development, physiology, and pathology. In common, MMP-9 has been recognized as a marker of various inflammatory diseases, such as atherosclerosis, arthritis, and systemic lupus erythematosus [37]. In addition, MMP-9 was closely associated with cell migration $[38,39]$. Several studies have particularly reported that MMP-2 and MMP-9 are involved in atherogenesis and play an important role in the stabilization of atherosclerotic plaques [40-42]. On this point, our results suggest that inhibitors of MMP-9 can offer promise as a therapeutic approach for the stabilization of vulnerable plaques. Moreover, recent investigations have demonstrated that wound healing in the skin is impaired by excessive activation of MMP-9 [43]. Therefore, it was suggested that fisetin also has potential as a therapeutic approach for healing in the skin. Future studies will be required to elucidate its detail on molecular mechanism.

A recent trend in the pharmaceutical industry is discovering strategies to develop the natural plant itself as the new drug. Indeed, two recent animal studies have demonstrated that LPS-induced acute pulmonary inflammation and collagen-induced arthritis were improved by fisetin treatment $[32,44]$. Another ex vivo study also demonstrated that fisetin was able to attenuate LPS-induced cytokine release from leukocytes of patients with chronic obstructive pulmonary disease or type 2 diabetes [45]. Many immunosuppressive drugs have been developed to attenuate inflammatory and autoimmune diseases [46-50]; nevertheless, most compounds exhibit significant side effects. Since polyphenols are commonly present in dietary fruits and vegetables, they presumably may be safer immunosuppressive agents [51, 52]. Fruits and vegetables contain a wide array of phytochemicals including sesquiterpene lactones, terpenoids, polysaccharides, and phenolic compounds. Our results, demonstrating the anti-inflammatory, antitumorigenic, and antioxidant effects of fisetin, strongly indicate that it can be developed as a new drug. Although fisetin is a promising candidate for immunotherapy, the potential of natural products containing fisetin as a therapeutic agent for a certain disease needs to be further verified in the clinical setting.

\section{Conclusions}

The present study demonstrated that fisetin attenuated the increments of inflammatory genes (MCP-1, IL-1b, and iNOS), the production of $\mathrm{NO}$, and the phosphorylation of MAPK, uPA, uPAR, and MMPs, induced by LPS in macrophages. These results suggested that fisetin may be a therapeutic agent for several macrophage-related diseases.

\section{Data Availability}

All material and data are present in the manuscript.

\section{Conflicts of Interest}

The authors declare no conflict of interest regarding this study.

\section{Acknowledgments}

We would like to thank Ms. Hiromi Tsuji for her technical assistance.

\section{References}

[1] A.'. al-Hrout, A. Chaiboonchoe, B. Khraiwesh et al., "Safranal induces DNA double-strand breakage and ER-stressmediated cell death in hepatocellular carcinoma cells," Scientific Reports, vol. 8, no. 1, p. 16951, 2018.

[2] A. A. Hamza, M. M. Ahmed, H. M. Elwey, and A. Amin, "Melissa officinalis protects against doxorubicin-induced cardiotoxicity in rats and potentiates its anticancer activity on MCF-7 cells," PLoS One, vol. 11, no. 11, article e0167049, 2016.

[3] A. Tresserra-Rimbau, E. B. Rimm, A. Medina-Remón et al., "Inverse association between habitual polyphenol intake and incidence of cardiovascular events in the PREDIMED study," Nutrition, Metabolism, and Cardiovascular Diseases, vol. 24, no. 6, pp. 639-647, 2014.

[4] A. Tresserra-Rimbau, E. B. Rimm, A. Medina-Remón et al., "Polyphenol intake and mortality risk: a re-analysis of the PREDIMED trial," BMC Medicine, vol. 12, no. 1, p. 77, 2014.

[5] E. Saito, M. Inoue, N. Sawada et al., "Association of coffee intake with total and cause-specific mortality in a Japanese population: the Japan public health center-based prospective study," The American Journal of Clinical Nutrition, vol. 101, no. 5, pp. 1029-1037, 2015.

[6] P. Mena, R. Domínguez-Perles, A. Gironés-Vilaplana, N. Baenas, C. García-Viguera, and D. Villaño, "Flavan-3-ols, anthocyanins, and inflammation," IUBMB Life, vol. 66, no. 11, pp. 745-758, 2014.

[7] E. Tili, J. J. Michaille, B. Adair et al., "Resveratrol decreases the levels of miR-155 by upregulating miR-663, a microRNA targeting JunB and JunD," Carcinogenesis, vol. 31, no. 9, pp. 1561-1566, 2010.

[8] M. E. Kim, J. Y. Na, and J. S. Lee, "Anti-inflammatory effects of trans-cinnamaldehyde on lipopolysaccharide-stimulated macrophage activation via MAPKs pathway regulation," Immunopharmacology and Immunotoxicology, vol. 40, no. 3, pp. 219224, 2018

[9] B. Amin and H. Hosseinzadeh, "Black cumin (Nigella sativa) and its active constituent, thymoquinone: an overview on the analgesic and anti-inflammatory effects," Planta Medica, vol. 82, no. 1-2, pp. 8-16, 2016.

[10] S. Zargar, N. J. Siddiqi, S. K. Al Daihan, and T. A. Wani, "Protective effects of quercetin on cadmium fluoride induced oxidative stress at different intervals of time in mouse liver," Acta Biochimica Polonica, vol. 62, no. 2, pp. 207-213, 2015.

[11] N. Alsalman, A. Aljafari, T. A. Wani, and S. Zargar, "Highdose aspirin reverses tartrazine-induced cell growth dysregulation independent of p53 signaling and antioxidant mechanisms in rat brain," BioMed Research International, vol. 2019, Article ID 9096404, 8 pages, 2019.

[12] M. Najafi, N. Hashemi Goradel, B. Farhood et al., "Macrophage polarity in cancer: a review," Journal of Cellular Biochemistry, vol. 120, no. 3, pp. 2756-2765, 2019.

[13] N. E. Thomford, D. A. Senthebane, A. Rowe et al., "Natural products for drug discovery in the 21st century: innovations for novel drug discovery," International Journal of Molecular Sciences, vol. 19, no. 6, p. 1578, 2018. 
[14] H. C. Pal, M. Athar, C. A. Elmets, and F. Afaq, "Fisetin inhibits UVB-induced cutaneous inflammation and activation of $\mathrm{PI} 3 \mathrm{~K} / \mathrm{AKT} / \mathrm{NF} \kappa \mathrm{B}$ signaling pathways in SKH-1 hairless mice," Photochemistry and Photobiology, vol. 91, no. 1, pp. 225-234, 2015.

[15] M. Gelderblom, F. Leypoldt, J. Lewerenz et al., "The flavonoid fisetin attenuates postischemic immune cell infiltration, activation and infarct size after transient cerebral middle artery occlusion in mice," Journal of Cerebral Blood Flow and Metabolism, vol. 32, no. 5, pp. 835-843, 2012.

[16] S. C. Kim, S. H. Kang, S. J. Jeong, S. H. Kim, H. S. Ko, and S. H. Kim, "Inhibition of c-Jun N-terminal kinase and nuclear factor $\kappa$ B pathways mediates fisetin-exerted anti-inflammatory activity in lipopolysccharide-treated RAW264.7 cells," Immunopharmacology and Immunotoxicology, vol. 34, no. 4, pp. 645-650, 2012.

[17] S. Y. Lyu and W. B. Park, "Production of cytokine and NO by RAW 264.7 macrophages and PBMC in vitro incubation with flavonoids," Archives of Pharmacal Research, vol. 28, no. 5, pp. 573-581, 2005.

[18] A. Hanneken, F. F. Lin, J. Johnson, and P. Maher, "Flavonoids protect human retinal pigment epithelial cells from oxidativestress-induced death," Investigative Ophthalmology \& Visual Science, vol. 47, no. 7, pp. 3164-3177, 2006.

[19] B. Sung, M. K. Pandey, and B. B. Aggarwal, "Fisetin, an inhibitor of cyclin-dependent kinase 6, down-regulates nuclear factor-kappaB-regulated cell proliferation, antiapoptotic and metastatic gene products through the suppression of TAK-1 and receptor-interacting protein-regulated IkappaBalpha kinase activation," Molecular Pharmacology, vol. 71, no. 6, pp. 1703-1714, 2007.

[20] Y. Suh, F. Afaq, N. Khan, J. J. Johnson, F. H. Khusro, and H. Mukhtar, "Fisetin induces autophagic cell death through suppression of mTOR signaling pathway in prostate cancer cells," Carcinogenesis, vol. 31, no. 8, pp. 14241433, 2010.

[21] T. Fotsis, M. S. Pepper, R. Montesano et al., "Phytoestrogens and inhibition of angiogenesis," Baillière's Clinical Endocrinology and Metabolism, vol. 12, no. 4, pp. 649-666, 1998.

[22] G. S. Prasath, S. I. Pillai, and S. P. Subramanian, "Fisetin improves glucose homeostasis through the inhibition of gluconeogenic enzymes in hepatic tissues of streptozotocin induced diabetic rats," European Journal of Pharmacology, vol. 740, pp. 248-254, 2014.

[23] A. Currais, C. Farrokhi, R. Dargusch et al., "Fisetin reduces the impact of aging on behavior and physiology in the rapidly aging SAMP8 mouse," The Journals of Gerontology. Series A, Biological Sciences and Medical Sciences, vol. 73, no. 3, pp. 299-307, 2018.

[24] J. J. Peterson, J. T. Dwyer, P. F. Jacques, and M. L. McCullough, "Associations between flavonoids and cardiovascular disease incidence or mortality in European and US populations," Nutrition Reviews, vol. 70, no. 9, pp. 491-508, 2012.

[25] S. M. Sendobry, J. A. Cornicelli, K. Welch, M. J. Grusby, and A. Daugherty, "Absence of T lymphocyte-derived cytokines fails to diminish macrophage 12/15-lipoxygenase expression in vivo," Journal of Immunology, vol. 161, pp. 1477-1482, 1998.

[26] V. Subramanian, H. A. Uchida, T. Ijaz, J. J. Moorleghen, D. A. Howatt, and A. Balakrishnan, "Calpain inhibition attenuates angiotensin II-induced abdominal aortic aneurysms and atherosclerosis in low-density lipoprotein receptor-deficient mice," Journal of Cardiovascular Pharmacology, vol. 59, no. 1, pp. 66-76, 2012.

[27] Y. Hada, H. A. Uchida, N. Otaka et al., "The protective effect of chlorogenic acid on vascular senescence via the Nrf2/HO-1 pathway," International Journal of Molecular Sciences, vol. 21, no. 12, p. 4527, 2020.

[28] H. A. Uchida, A. Poduri, V. Subramanian, L. A. Cassis, and A. Daugherty, "Urokinase-type plasminogen activator deficiency in bone marrow-derived cells augments rupture of angiotensin II-induced abdominal aortic aneurysms," Arteriosclerosis, Thrombosis, and Vascular Biology, vol. 31, no. 12, pp. 2845-2852, 2011.

[29] M. Okuyama, H. A. Uchida, Y. Hada et al., "Exogenous vasohibin-2 exacerbates angiotensin II-induced ascending aortic dilation in mice," Circulation Reports, vol. 1, no. 4, pp. 155$161,2019$.

[30] R. Umebayashi, H. A. Uchida, Y. Kakio, V. Subramanian, A. Daugherty, and J. Wada, "Cilostazol attenuates angiotensin II-induced abdominal aortic aneurysms but not atherosclerosis in apolipoprotein E-deficient mice," Arteriosclerosis, Thrombosis, and Vascular Biology, vol. 38, no. 4, pp. 903912, 2018.

[31] K. Yao, L. Zhang, Y. Zhang, P. Ye, and N. Zhu, “The flavonoid, fisetin, inhibits UV radiation-induced oxidative stress and the activation of NF-kappaB and MAPK signaling in human lens epithelial cells," Molecular Vision, vol. 14, pp. 1865-1871, 2008.

[32] J. D. Lee, J. E. Huh, G. Jeon et al., "Flavonol-rich RVHxR from Rhus verniciflua Stokes and its major compound fisetin inhibits inflammation-related cytokines and angiogenic factor in rheumatoid arthritic fibroblast-like synovial cells and in vivo models," International Immunopharmacology, vol. 9, no. 3, pp. 268-276, 2009.

[33] A. A. Hamza, G. H. Heeba, S. Hamza, A. Abdalla, and A. Amin, "Standardized extract of ginger ameliorates liver cancer by reducing proliferation and inducing apoptosis through inhibition oxidative stress/inflammation pathway," Biomedicine \& Pharmacotherapy, vol. 134, article 111102, 2021.

[34] S. M. El-Dakhly, A. A. A. Salama, S. O. M. Hassanin, N. N. Yassen, A. A. Hamza, and A. Amin, "Aescin and diosmin each alone or in low dose-combination ameliorate liver damage induced by carbon tetrachloride in rats," BMC Research Notes, vol. 13, no. 1, p. 259, 2020.

[35] S. H. Liu, C. H. Lin, S. K. Hung, J. H. Chou, C. W. Chi, and S. L. $\mathrm{Fu}$, "Fisetin inhibits lipopolysaccharide-induced macrophage activation and dendritic cell maturation," Journal of Agricultural and Food Chemistry, vol. 58, no. 20, pp. 10831-10839, 2010.

[36] R. H. Chou, S. C. Hsieh, Y. L. Yu, M. H. Huang, Y. C. Huang, and Y. H. Hsieh, "Fisetin inhibits migration and invasion of human cervical cancer cells by down-regulating urokinase plasminogen activator expression through suppressing the p38 MAPK-dependent NF- $\kappa$ B signaling pathway," PLoS One, vol. 8, no. 8, article e71983, 2013.

[37] G. V. Halade, Y. F. Jin, and M. L. Lindsey, "Matrix metalloproteinase (MMP)-9: a proximal biomarker for cardiac remodeling and a distal biomarker for inflammation," Pharmacology \& Therapeutics, vol. 139, no. 1, pp. 32-40, 2013.

[38] Y. H. Kim, H. J. Kwon, and D. S. Kim, "Matrix metalloproteinase 9 (MMP-9)-dependent processing of $\beta$ ig-h3 protein regulates cell migration, invasion, and adhesion," The Journal of Biological Chemistry, vol. 287, no. 46, pp. 38957-38969, 2012. 
[39] Y. Gong, E. Hart, A. Shchurin, and J. Hoover-Plow, "Inflammatory macrophage migration requires MMP-9 activation by plasminogen in mice," The Journal of Clinical Investigation, vol. 118, no. 9, pp. 3012-3024, 2008.

[40] P. J. Gough, I. G. Gomez, P. T. Wille, and E. W. Raines, "Macrophage expression of active MMP-9 induces acute plaque disruption in apoE-deficient mice," The Journal of Clinical Investigation, vol. 116, no. 1, pp. 59-69, 2006.

[41] Y. Z. Kong, X. Yu, J. J. Tang et al., "Macrophage migration inhibitory factor induces MMP-9 expression: implications for destabilization of human atherosclerotic plaques," Atherosclerosis, vol. 178, no. 1, pp. 207-215, 2005.

[42] D. Wågsäter, C. Zhu, J. Björkegren, J. Skogsberg, and P. Eriksson, "MMP-2 and MMP-9 are prominent matrix metalloproteinases during atherosclerosis development in the Ldlr(-/-)Apob(100/100) mouse," International Journal of Molecular Medicine, vol. 28, no. 2, pp. 247-253, 2011.

[43] L. Zhou, M. Ren, T. Zeng et al., “TET2-interacting long noncoding RNA promotes active DNA demethylation of the MMP-9 promoter in diabetic wound healing," Cell Death \& Disease, vol. 10, no. 11, p. 813, 2019.

[44] L. Geraets, A. Haegens, K. Brauers et al., "Inhibition of LPSinduced pulmonary inflammation by specific flavonoids," Biochemical and Biophysical Research Communications, vol. 382, no. 3, pp. 598-603, 2009.

[45] A. R. Weseler, L. Geraets, H. J. Moonen et al., "Poly (ADPribose) polymerase-1-inhibiting flavonoids attenuate cytokine release in blood from male patients with chronic obstructive pulmonary disease or type 2 diabetes," The Journal of Nutrition, vol. 139, no. 5, pp. 952-957, 2009.

[46] H. Hackstein, A. E. Morelli, A. T. Larregina et al., “Aspirin inhibits in vitro maturation and in vivo immunostimulatory function of murine myeloid dendritic cells," Journal of Immunology, vol. 166, no. 12, pp. 7053-7062, 2001.

[47] A. Mehling, S. Grabbe, M. Voskort, T. Schwarz, T. A. Luger, and S. Beissert, "Mycophenolate mofetil impairs the maturation and function of murine dendritic cells," Journal of Immunology, vol. 165, no. 5, pp. 2374-2381, 2000.

[48] L. Piemonti, P. Monti, P. Allavena et al., "Glucocorticoids affect human dendritic cell differentiation and maturation," Journal of Immunology, vol. 162, pp. 6473-6481, 1999.

[49] K. Steinbrink, M. Wölfl, H. Jonuleit, J. Knop, and A. H. Enk, "Induction of tolerance by IL-10-treated dendritic cells," Journal of Immunology, vol. 159, pp. 4772-4780, 1997.

[50] Y. Yamaguchi, H. Tsumura, M. Miwa, and K. Inaba, "Contrasting effects of TGF-beta 1 and TNF-alpha on the development of dendritic cells from progenitors in mouse bone marrow," Stem Cells, vol. 15, no. 2, pp. 144-153, 1997.

[51] H. Kamal, S. Jafar, P. Mudgil, C. Murali, A. Amin, and S. Maqsood, "Inhibitory properties of camel whey protein hydrolysates toward liver cancer cells, dipeptidyl peptidaseIV, and inflammation," Journal of Dairy Science, vol. 101, no. 10, pp. 8711-8720, 2018.

[52] H. Ashktorab, A. Soleimani, G. Singh et al., "Saffron: the golden spice with therapeutic properties on digestive diseases," Nutrients, vol. 11, no. 5, p. 943, 2019. 